\title{
Upregulation of miR-20a and miR-106b is involved in the acquisition of malignancy of pediatric brainstem gliomas
}

\author{
XUAN WANG $^{1 *}$, HONGWEI ZHANG $^{1 *}$, ANLING ZHANG $^{2-3}$, LEI HAN ${ }^{2-3}$, KUN WANG $^{4}$, RAN LIU $^{5}$, \\ SHAOHUA YANG ${ }^{5}$, PEIYU PU ${ }^{2-3}$, CHANGHONG SHEN $^{2}$, CHUNSHENG KANG $^{2-3}$ and CHUNJIANG YU ${ }^{1}$ \\ ${ }^{1}$ Department of Neurosurgery, Beijing Sanbo Brain Hospital, Capital Medical University, Beijing 100093; \\ ${ }^{2}$ Department of Neurosurgery, Tianjin Medical University General Hospital, Tianjin 300052; ${ }^{3}$ Laboratory of Neuro- \\ Oncology, Tianjin Neurological Institute, Tianjin 300052; ${ }^{4}$ Department of Neurosurgery, Sir Run Run Show \\ Hospital, School of Medicine, Zhejiang University, Hangzhou, Zhejiang 310016, P.R. China; ${ }^{5}$ Department of \\ Pharmacology and Neuroscience, University of North Texas Health Science Center, Fort Worth, TX 76107, USA
}

Received May 17, 2012; Accepted June 22, 2012

DOI: $10.3892 /$ or.2012.1927

\begin{abstract}
Brainstem glioma (BSG) is an entity which commonly occurs in pediatric patients and carries a dismal prognosis. However, the category of adult BSG has displayed considerably benign biological behavior, thus providing a unique perspective to comparatively understand the malignant features of pediatric BSG. microRNAs (miRNAs) have been associated with cancer development and progression. To identify miRNAs specifically involved in the acquisition of malignant progression of pediatric BSG, we analyzed the miRNA expression profiles in orthotopic models which could simulate the BSG heterogeneity by microarrays. Our research revealed that miR-20a and miR-106b (known to be closely related) were the two most robust upregulated miRNAs in pediatric BSG compared to adult subtype. Furthermore, the two types of human BSG tissue were utilized to verify the
\end{abstract}

Correspondence to: Professor Chunjiang Yu, Department of Neurosurgery, Beijing Sanbo Brain Hospital, Capital Medical University, 50 Xiangshan-Yikesong Road, Haidian District, Beijing 100093, P.R. China

E-mail: cjyu1955@sina.com

Professor Chunsheng Kang, Laboratory of Neuro-Oncology, Tianjin Neurological Institute, Tianjin 300052, P.R. China

E-mail: osen1984@gmail.com

*Contributed equally

Abbreviations: BSG, brain stemglioma; CNS, central nervous system; miRNA, microRNA; FCAbsolute, absolute value of the fold-change; 3' UTR, 3' untranslated region; WHO, World Health Organization; mRNA, messenger RNA; Cy3, Cyanine3-pCp; FISH, fluorescence in situ hybridization

Key words: miR-20a, miR-106b, microRNA, brainstem glioma, pediatric tumor microarray data by qRT-PCR and in situ hybridization. The results indicated good consistency with that of the microarray method. In conclusion, our studies provide evidence that miR-20a and miR-106b may serve as putative causative involvement of malignant progression of pediatric BSG, thereby serving as likely novel targets for constraining the rapid fatal course of pediatric BSG.

\section{Introduction}

Pediatric brainstem glioma (BSG) is now the main cause of death in pediatric patient with solid tumors, which constitute $15-20 \%$ of all central nervous system (CNS) neoplasms that developed in childhood (1). In particular, typical pediatric BSG is characterized by malignant feature, about $80 \%$ of these tumors are 'diffuse intrinsic' or 'diffusely infiltrative' category, leading to diffuse increase of the brainstem and making complete resection nearly impossible (2). Despite recent attempts to optimize or combine radiotherapy and chemotherapy, the poor prognosis of these tumors has not changed in the past two decades (3). These circumstances leave the median survival after diagnosis at less than 1 year (1).

Although adult intracranial gliomas account for more than $40 \%$ of all intracranial tumors $(4,5)$, it is noteworthy that adult patients with BSG are extremely rare and accounts for less than $2 \%$ of adult gliomas (6-8). Moreover, compared to pediatric BSG, adult BSG is typically much less invasive, and there is a clear and intact boundary between the BSG and surrounding tissue (9). Thus, the therapeutic effect of surgery is better. Very different from pediatric BSG, adult subtype has a median survival period up to 7.3 years after diagnosis (6). These and other data suggest that BSG is a unique glioma subtype with biological and molecular pathology mechanisms different from common gliomas (6). Discerning the key differences between pediatric and adult BSG may prove indispensable to the fight against pediatric BSG (10), a fight that has not yielded many positive victories in recent years (1).

Molecular genetic analysis will provide insight into gliomagenesis, but the precise pattern of genetic abnormalities 
within the group of pediatric BSG is still poorly documented. Existing researches on this issue were fragmented and limited in scope (11). Nevertheless, to our knowledge, no study has examined the molecular mechanisms of BSG malignant progression from the aspect of the striking difference between pediatric and adult types of BSG. Also, we speculate that the central role of miRNA in modulating gene expression will present a comprehensive perspective for understanding the molecular bases of pediatric BSG.

microRNAs (miRNAs) are a set of small non-coding RNA molecules (12). Partial complementary pairing between the 3'-untranslated region (3'UTR) of the messenger RNA (mRNA) and the 5' seed region of a miRNA is essential for the post-transcriptional modulation of the target gene expression (13). Given the diversity and abundance of target genes, miRNAs appear to functionally interact with various components of cellular signal transduction pathways known as tumor suppressor genes or oncogenes to generate a complex combinatorial network $(14,15)$, suggesting that miRNAs play central role in carcinogenesis (16).

We previously reproduced the major characteristics of two kinds of human BSG in rat orthotopic C6 cell BSG models, respectively (10). In the current study, we modified the former models, replaced by athymic rats using suspensions of human malignant glioma cell line. With the modified model, we examined, for the first time, the expression profile of aberrant miRNAs of pediatric BSG compared to adult type. Human BSG tissues were utilized to verify the microarray results by qRT-PCR and in situ hybridization.

\section{Materials and methods}

Cell culture, experimental animals and model making. The LN229 cell line was obtained from the China Academia Sinica Cell Repository. All cells were maintained in Dulbecco's modified Eagle's medium (DMEM, Gibco) supplemented with $10 \%$ fetal bovine serum (Gibco), at $37^{\circ} \mathrm{C}$ with $5 \% \mathrm{CO}_{2}$ in a humidified incubator.

Pediatric male athymic rats (3-week old, body weight $45 \pm 5 \mathrm{~g}$ ) and adult male athymic rats (10-week old, body weight $275 \pm 50 \mathrm{~g}$ ) were purchased from the Cancer Institute of The Chinese Academy of Medical Science. All animal procedures were approved by the Capital Medical University Animal Care and Use Committee.

The BSG model was modified from our previous description (10). In detail, animals were anesthetized with $10 \%$ chloral hydrate administered intraperitoneally, and then positioned in a stereotaxic frame. A midline incision was made and the lambda suture was identified. A bur hole was drilled $0.5 \mathrm{~mm}$ to the right of and $1 \mathrm{~mm}$ posterior to the lambda, taking care to keep the integrity of the dura mater. The LN229 cells were trypsinized, washed twice, resuspended, and diluted in DMEM (without FBS) to a concentration of $2.0 \times 10^{5}$ cells $/ 15 \mu 1$. Using a Hamilton syringe with a 27 -gauge needle, LN229 cells were then implanted into the pons $(15 \mu 1,10-\mathrm{mm}$ depth from the dura mater in adult rats and $5 \mu 1,7 \mathrm{~mm}$ in young rats). All rats were assessed by MRI (Philips Achieva 1.5T Nova).

Tissue preparation and RNA extraction. After anesthesia and transcardial perfusion with $4 \%$ paraformaldehyde (to elimi- nate some of the red blood cells), the animals were killed by decapitation. Immediately the brain tissue was isolated, the edema and normal brain tissue around the tumor focus tissue was strictly selected, the cerebral pia mater was cleared and blood vessels were removed under a microscope, quickly stored into liquid nitrogen or disruption. Each frozen tissue piece was homogenized in $1 \mathrm{ml}$ TRIzol reagent (Invitrogen) for RNA extraction, as previously described (17).

miRNA microarray analysis. The RNA was purified from total RNA using a mirVana miRNA isolation kit (AM1561, Ambion). The miRNA expression profiles were performed at Beijing CapitalBio Corp. using miRNA Microarray system (Version 2.2) with Agilent miRNA Complete Labeling and Hyb kit (p/n 5190-0456), containing 723 human and 76 human viral miRNAs catalogued in the Sanger miRNA database v10.1 (Agilent Technologies). Purified RNA quality was verified using the 2100 Bioanalyzer (Agilent Technologies). With a sample input of $100 \mathrm{ng}$, purified RNA was treated with calf intestinal alkaline phosphatase (CIP) for 5' end dephosphorylation, and then labeled with Cyanine3-pCp (Сy3) using T4 RNA Ligase to the 3' end of RNA. The generated fluorescent miRNA was hybridized to the microarray probes at $55^{\circ} \mathrm{C}$ for $20 \mathrm{~h}$. The microarray slide was subsequently washed and scanned using the Agilent scanner to obtain microarray images.

Microarray data analyses. The microarray image information was converted into spot intensity values using Scanner Control Software Rev 7.0 (Agilent Technologies). The signal after background subtraction was exported directly into the GeneSpring GX10 software (Agilent Technologies) for quantile normalization. The mean normalized signal from biological replicates was used for comparative expression analysis. Unpaired t-test with Benjamini-Hochberg correction was performed to determine differentially expressed miRNAs between pediatric and adult BSG subtypes. The fold-changes ( $\log 2$ transformed) of expression signals between pediatric and adult BSG subtypes were calculated from the normalized values. Hierarchical clustering was performed with Pearson correlation using Cluster 3.0 software by Average linkage clustering. Dendrograms and expression pattern maps were generated by TreeView software from the Stanford University.

Patients and specimens. The study was performed according to a protocol approved by the Capital Medical University Ethics Committee. Human BSG tissue samples were obtained from Sanbo Brain Hospital affiliated to Capital Medical University after informed consent from patients diagnosed with BSG. One WHO-I and 1 WHO-II adult BSG, 1 WHO-III and 2 WHO-IV pediatric BSG tissues were resected during surgery and immediately frozen in liquid nitrogen for subsequent total RNA extraction.

Quantitative RT-PCR ( $q R T-P C R)$. The expression levels of specific miRNAs were validated by quantitative RT-PCR using the Hairpin-it ${ }^{\text {TM }}$ miRNAs qPCR Quantitation kit (GenePharma Co., Ltd., Shanghai, China). Briefly, the $20 \mu \mathrm{l}$ reverse transcriptase reactions included purified total RNA (2-10 ng per reaction) were incubated in a PTC-200 thermal 


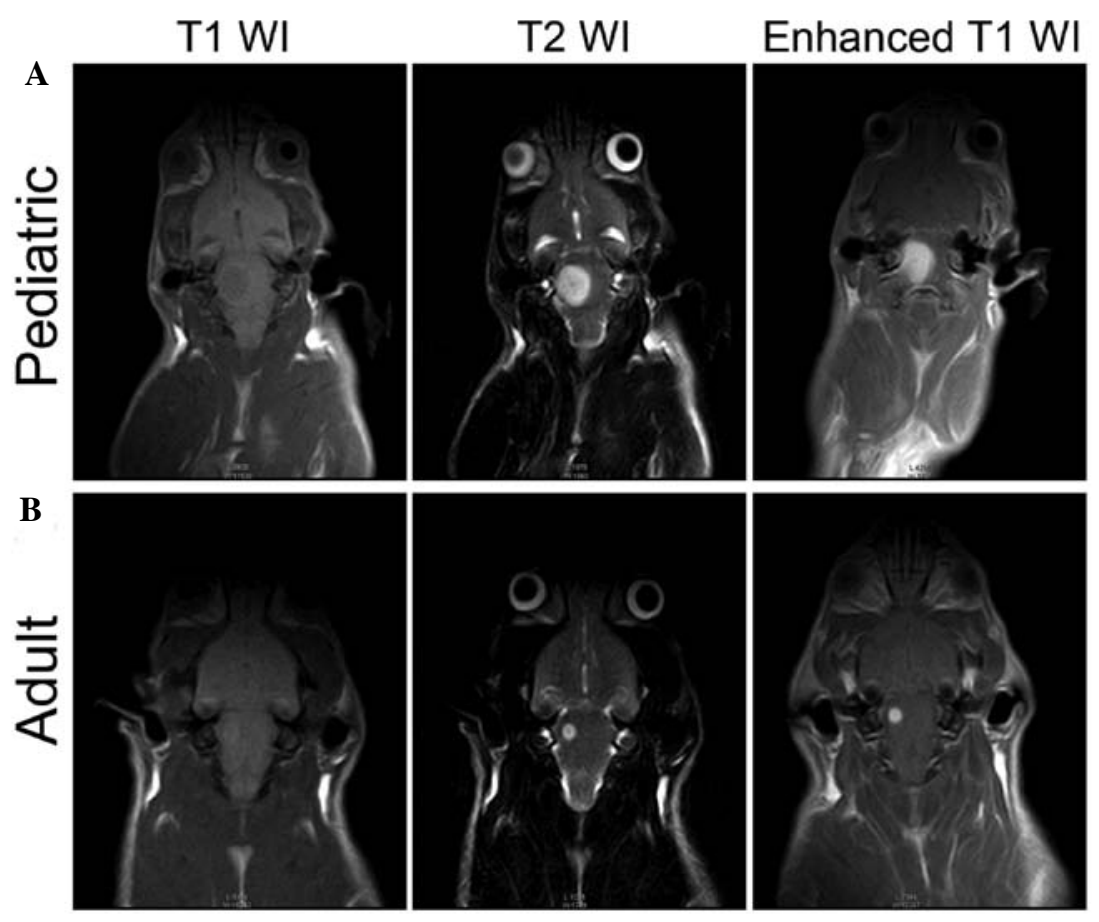

Figure 1. Pediatric BSG and adult BSG orthotopic models imaging by MRI scan. The orthotopically injected tumor into the pons was imaged after 2 weeks of tumor growth. (A) Pediatric subtype; (B) adult subtype.

cycler (Bio-Rad Laboratories, Hercules, CA, USA) in a 96-well plate for $30 \mathrm{~min}$ at $16^{\circ} \mathrm{C}$, followed by $30 \mathrm{~min}$ at $42^{\circ} \mathrm{C}, 10 \mathrm{~min}$ at $85^{\circ} \mathrm{C}$, and then held at $4^{\circ} \mathrm{C}$. All reverse transcriptase reactions, including no-template controls and RT minus controls, were run in duplicate. Real-time PCR was performed using a DNA Engine Opticon 2 system (Bio-Rad Laboratories). The following real-time PCR protocol was used: denaturation program $\left(95^{\circ} \mathrm{C}\right.$ for $\left.3 \mathrm{~min}\right)$, amplification and quantification program ( $40 \mathrm{cycles}$ of $95^{\circ} \mathrm{C}$ for $12 \mathrm{sec}$ and $62^{\circ} \mathrm{C}$ for $40 \mathrm{sec}$ ), and melting curve program (from $62^{\circ} \mathrm{C}$ to $95^{\circ} \mathrm{C}$, read every $0.2^{\circ} \mathrm{C}$, hold $2 \mathrm{sec}$ ). U6 RNA (GenePharma) was used for normalization. Data are shown as fold-change $\left(2^{-\Delta \Delta C T}\right)$.

Fluorescence in situ hybridization (FISH). To study the spatial and temporal expression of miRNAs with high sensitivity and resolution, FISH was performed with in situ hybridization kit (Boster, Wuhan, China). LNA/DNA oligos had the following sequences: LNA-miR-106b 5'-ATC TGC ACT GTC AGC ACT TTA-3', LNA-miR-20a 5'-CTA CCT GCA CTA TAA GCA CTT TA-3', scramble 5'-GTG TAA CAC GTC TAT ACG CCC A-3', U6 5'-CAC GAA TTT GCG TGT CAT CCT T-3'. DEPC water $(0.1 \%)$ was used for all solutions and appliances necessary for FISH. After deparaffinized and deproteinated, the tissue sections were pre-hybridized for $4 \mathrm{~h}$ at $42^{\circ} \mathrm{C}$, followed by incubation with LNA-miRNA hybridization solution (containing probe) overnight at $42^{\circ} \mathrm{C}$. Then the sections were washed with gradient SSC thoroughly (2X SSC, 0.5X SSC and $0.2 \mathrm{X} \mathrm{SSC}$ ) to remove the background signals, followed by treatment with biotinylated digoxin antibody at $37^{\circ} \mathrm{C}$ for $1 \mathrm{~h}$, and Cy3-avidin (used to label miRNA) at $37^{\circ} \mathrm{C}$ for $30 \mathrm{~min}$. Nuclei were counterstained with DAPI, then sections were detected under FV1000 fluorescence microscope (Olympus, Tokyo, Japan) and analyzed using IPP5.1 (Olympus).

\section{Results}

Assessment of pediatric BSG and adult BSG orthotopic models. The orthotopic models simulating the BSG heterogeneity were modified from our previous description (10). The orthotopically injected LN229 cells into the pons of animals was imaged after 2 weeks of tumor growth. The MRI scan revealed that human glioma cells in the brainstems of pediatric group progress more extensively than in the adult group. They grew rapidly and enlarged to occupy most parts of the pons of pediatric group (Fig. 1A). In adult group, the lesions grew relatively focally (Fig. 1B). These results suggest that the quality of the models we developed meet the requirements for subsequent study.

The microarray analysis for the expression profile of aberrant miRNAs of pediatric BSG compared to adult type in orthotopic models. To identify miRNAs specifically involved in the acquisition of malignant progression of pediatric BSG, we employed a microarray to analyze the miRNA expression profiles in the two groups of orthotopic models which could simulate the BSG heterogeneity. Two hundred and sixteen miRNAs were detected in both the pediatric BSG group and the adult BSG group (Fig. 2A).

The data from the pediatric BSG group are compared with the data of adult BSG group to discover the differential expressed miRNAs. Those miRNAs with FCAbsolute $>3.5$ and corrected P-value $<0.02$ were identified as exhibiting considerable upregulated expression, or, they may be considered as exhibiting considerable downregulated expression likewise.

With these criteria, our research revealed 39 miRNAs to be differentially expressed in the pediatric BSG group vs. adult group, including 10 upregulated expression miRNAs and 
Table I. Significantly changed miRNAs with corrected P-value and fold-change (absolute) by comparison between pediatric BSG and adult BSG in orthotopic models.

\begin{tabular}{lll}
\hline miRNA ID & FCAbsolute & Corrected P-value \\
\hline Positively correlated miRNAs & \\
hsa-miR-20a & 65.632 & $4.93 \mathrm{E}-05$ \\
hsa-miR-106b & 44.682 & $4.93 \mathrm{E}-05$ \\
hsa-miR-146a & 25.36153 & $4.93 \mathrm{E}-05$ \\
hsa-miR-34a & 15.55422 & $1.26 \mathrm{E}-04$ \\
hsa-miR-140-3p & 13.98039 & 0.0011711 \\
hsa-miR-140-5p & 13.59603 & $4.65 \mathrm{E}-04$ \\
hsa-miR-93 & 10.21174 & $7.77 \mathrm{E}-04$ \\
hsa-miR-363 & 9.115072 & 0.0076257 \\
hsa-miR-196b & 3.546545 & 0.0030593 \\
hsa-miR-18b & 3.512272 & 0.0030655 \\
Negatively correlated miRNAs & \\
hsa-miR-224 & 12.30044 & $5.50 \mathrm{E}-04$ \\
hsa-miR-22 & 7.664119 & $2.87 \mathrm{E}-04$ \\
hsa-miR-1260b & 6.506731 & 0.0076637 \\
hsa-miR-9* & 7.519141 & $4.93 \mathrm{E}-05$ \\
hsa-miR-629* & 6.106636 & 0.01767 \\
hsa-miR-9 & 6.086732 & 0.0019944 \\
hsa-miR-135a* & 5.71923 & $3.92 \mathrm{E}-04$ \\
hsa-miR-542-5p & 5.628573 & $7.38 \mathrm{E}-04$ \\
hsa-miR-663 & 4.729385 & 0.0084151 \\
hsa-miR-29b & 5.151732 & $5.50 \mathrm{E}-04$ \\
hsa-miR-424 & 4.837116 & 0.0027836 \\
hsa-miR-193b & 4.67872 & 0.0036245 \\
hsa-miR-130a & 4.44713 & 0.0010245 \\
hsa-miR-99a & 4.273679 & $5.50 \mathrm{E}-04$ \\
hsa-miR-29c & 4.105427 & $8.15 \mathrm{E}-04$ \\
hsa-let-7c & 3.908946 & 0.0015876 \\
hsa-miR-134 & 3.791241 & $3.92 \mathrm{E}-04$ \\
hsa-miR-1274a & 3.634825 & 0.0070172 \\
hsa-miR-29a & 3.613525 & 0.0015876 \\
hsa-let-7b & 3.535912 & 0.0035764 \\
\hline
\end{tabular}

29 downregulated expression miRNAs (Table I, Fig. 2A). hsa-miR-20a, hsa-miR-106b, hsa-miR-146a and hsamiR-34a, were found to be the most significantly upregulated miRNAs, while hsa-miR-224, hsa-miR-22, hsa-miR-1260b and hsa-miR-9*, to be the most significantly downregulated ones.

Quantitative RT-PCR validation of miR-20a and miR-106b. Amongst the aberrantly expressed miRNAs according to microarray analysis, the most considerable fold-changes are seen in miR-20a and miR-106b (Table I, Fig. 2B). Specifically, miR-106b belongs to miR-106b-25 cluster, whereas its paralog miR-17-92 cluster contains miR-20a. These findings indicate that miR-20a and miR-106b might be putative causative miRNAs, hence, chosen as candidate for validation in human BSG to confirm the result obtained from orthotopic models. Thus, we employed qRT-PCR on human specimens of 3 pediatric BSG and 2 adult BSG. qRT-PCR analysis verified the data screened using microarray: miR-20a and miR-106b expression patterns behaved similarly in human specimens, both levels were markedly higher in pediatric BSG in comparison with adult ones, though the extent was slightly lower than the data revealed by orthotopic models (Tables II and III). There were no significant differences between miR-20a or miR-106b levels in grade III and grade IV pediatric BSG (Tables IV and V), or grade I and grade II adult BSG (Tables VI and VII).

Fluorescence in situ hybridization validation of miR-20a and $m i R-106 b$. To more firmly identify the changes of miRNA expression obtained from orthotopic models, FISH was further applied to localize the expression patterns of miR-20a and miR-106b using LNA probes. As expected, the staining pattern of sections from human pediatric BSG specimens were particularly striking, whereas low in situ signals observed in adult ones, concurred with our qRT-PCR data (Fig. 3).

\section{Discussion}

Brainstem glioma (BSG) is an entity which commonly occurs in pediatric patients $(1,18)$. The benign property of adult BSG has provided a unique perspective to comparatively understand pediatric BSG (10). Nevertheless, BSG in adults are poorly understood because they are quite unusual (6). Besides the distinction in incidence, biological behavior, invasion pattern, prognosis and treatment outcome, many studies indicated the concept that these tumors form a homogeneous group have changed, and now it should be recognized that BSGs are a heterogenous group with differing genetic characteristics (10).

Many clinical reports have shown that the majority of pediatric BSG are high grade gliomas (WHO grades III and IV) revealed by histopathological examination $(2,19,20)$. Correspondingly, Guillamo et al have shown that benign histology (WHO grades I and II) was found in up to $82 \%$ in adult group (6). Moreover, in the previous study we also observed that despite the application the suspension of C6 cells was identical, after the establishment of orthotopic BSG model, malignant grade of pediatric BSG was significantly higher than the adult group. A huge degree of difference in cell proliferation and apoptosis has also been reported (10). In addition, a retrospective analysis was undertaken on 40 BSG patients (10 children and 30 adults) with the identical malignant grade (WHO II), median survival was 35.2 months in the adult group, having far better prognosis than the pediatric patients with the survival of 16.5 months (21). Therefore, in the current study, we performed genetic analysis of pediatric BSG compared to adult type by miRNA microarrays to see if there were any genetic characteristics that could afford relevant information in terms of management of patients and etiology of pediatric BSG.

Indeed, existing research on the oncogenic role of miRNAs in brain tumors has achieved some remarkable results (22), such as pituitary adenoma (23), and medulloblastoma (24). Ciafrè et al (25) profiled the expression of 245 miRNAs in 


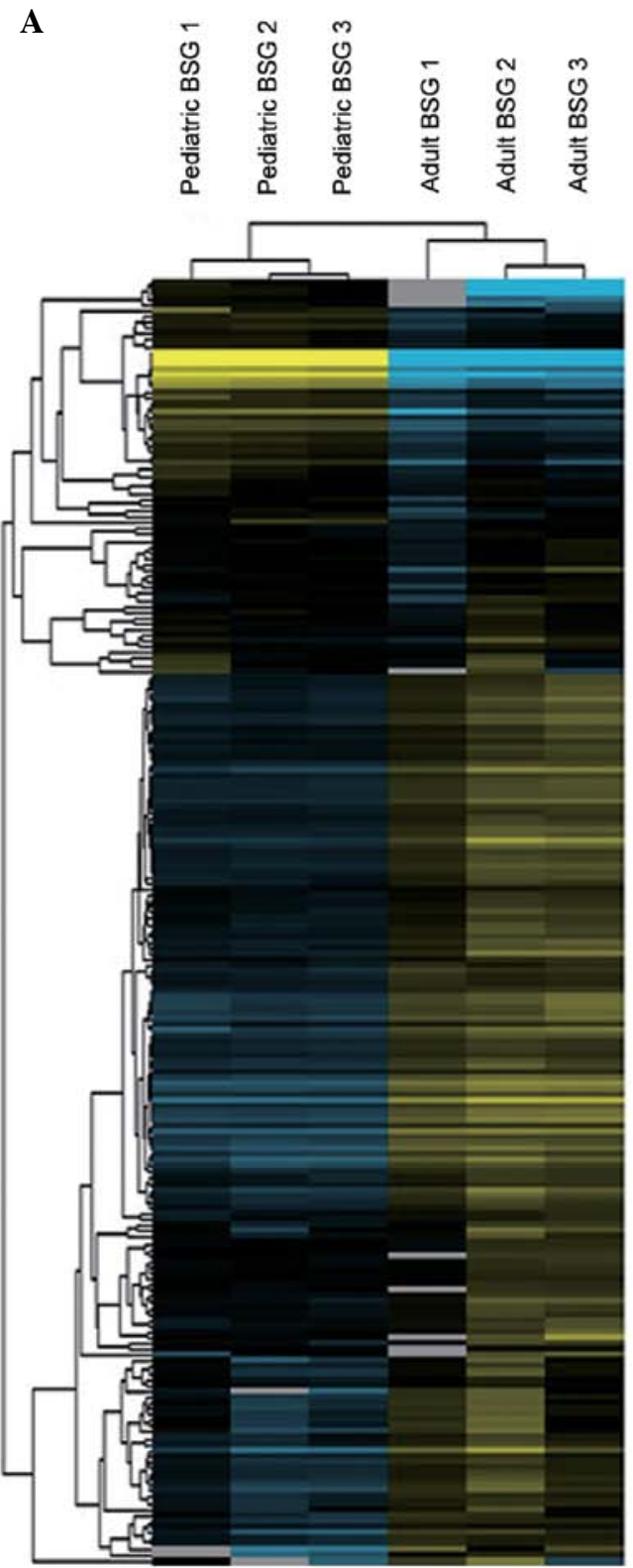

B

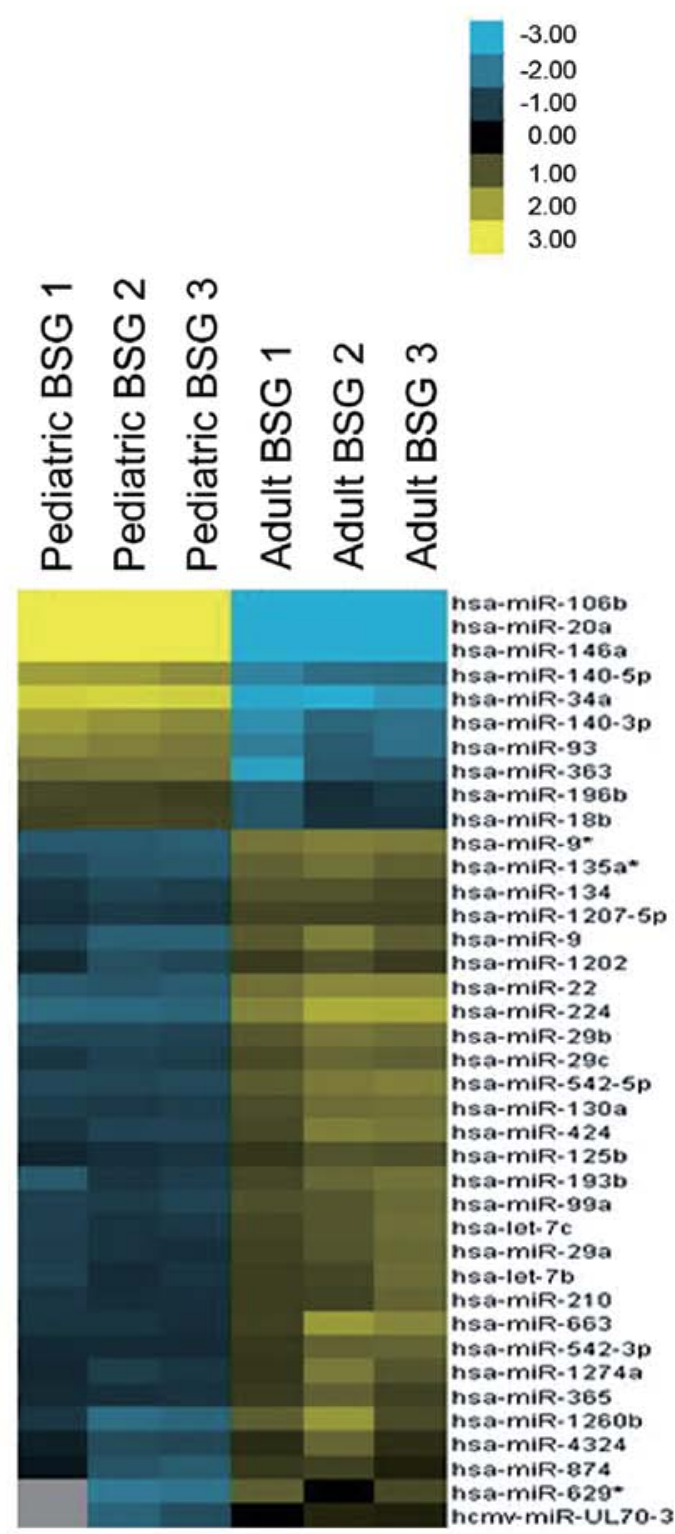

Figure 2. Hierarchical cluster analysis of 6 sets of array data in the pediatric BSG and adult BSG orthotopic models. Colors indicate relative signal intensities. Yellow squares represent the positive values (upregulation), black squares represent zero (no change), and blue squares represent negative values (downregulation). (A) All the detected 216 miRNAs in the pediatric BSG and adult BSG orthotopic models. (B) The differentially-expressed miRNAs in pediatric BSG compared to adult BSG orthotopic models.

Table II. The expression changes of miR-20a between human specimens of adult and pediatric BSG.

\begin{tabular}{lcccr}
\hline Group & Specimen & $2^{-\Delta \text { СT }}$ & RQ (fold) & P-value \\
\hline Adult & 2 & $9.88 \pm 0.460521444$ & 1 & $<0.01$ \\
Pediatric & 3 & $6.59 \pm 0.18594354$ & $9.46072 \pm 0.37015$ & $<$ \\
\hline
\end{tabular}

Table III. The expression changes of miR-106b between human specimens of adult and pediatric BSG.

\begin{tabular}{lcccr}
\hline Group & Specimen & $2^{-\Delta C T}$ & RQ (fold) & P-value \\
\hline Adult & 2 & $8.51500 \pm 0.21380$ & 1 & \\
Pediatric & 3 & $5.09111 \pm 0.42923$ & $11.04985 \pm 0.92818$ & $<0.01$ \\
\hline
\end{tabular}


Table IV. The expression changes of miR-20a between human specimens of adult BSGs with different WHO classification.

\begin{tabular}{lcccc}
\hline Group & Specimen & $2^{-\Delta \mathrm{CT}}$ & RQ (fold) & P-value \\
\hline Adult WHO-I & 1 & $9.79667 \pm 0.58731$ & 1 & $>0.90668 \pm 0.19905$ \\
Adult WHO-II & 1 & $9.96333 \pm 0.40550$ & $>0.01$ \\
\hline
\end{tabular}

Table V. The expression changes of miR-106b between human specimens of adult BSGs with different WHO classification.

\begin{tabular}{lcccc}
\hline Group & Specimen & $2^{-\Delta \mathrm{CT}}$ & RQ (fold) & P-value \\
\hline Adult WHO-I & 1 & $8.56333 \pm 0.18502$ & 1 & $1.07978 \pm 0.18849$ \\
Adult WHO-II & 1 & $8.46667 \pm 0.27025$ & $>0.01$ \\
\hline
\end{tabular}

Table VI. The expression changes of miR-20a between human specimens of pediatric BSGs with different WHO classification.

\begin{tabular}{lcccc}
\hline Group & Specimen & $2^{-\Delta \mathrm{CT}}$ & RQ (fold) & P-value \\
\hline Pediatric WHO-III & 1 & $6.56667 \pm 0.19732$ & 1 & $0.97748 \pm 0.05130$ \\
Pediatric WHO-IV & 2 & $6.60167 \pm 0.19813$ & $>0.01$ \\
\hline
\end{tabular}

Table VII. The expression changes of miR-106b between human specimens of pediatric BSGs with different WHO classification.

\begin{tabular}{lcccc}
\hline Group & Specimen & $2^{-\Delta \mathrm{CT}}$ & RQ (fold) & P-value \\
\hline Pediatric WHO-III & 1 & $5.15000 \pm 0.42930$ & 1 & $1.07915 \pm 0.10707$ \\
Pediatric WHO-IV & 2 & $5.06167 \pm 0.46684$ & $>0.01$ \\
\hline
\end{tabular}

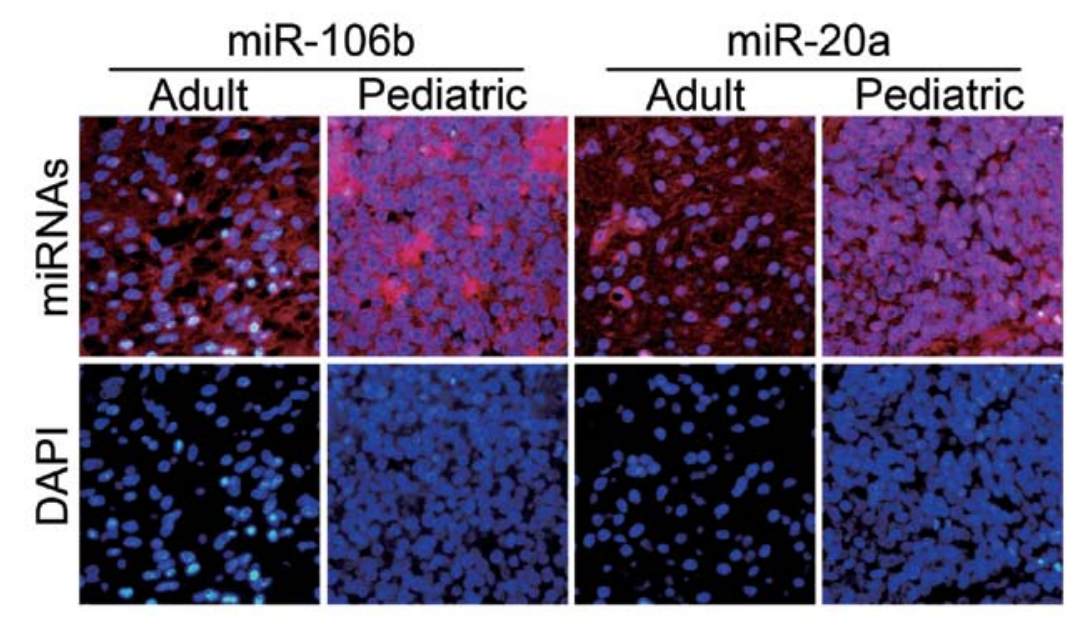

Figure 3. FISH detection of miR-106b and miR-20a in between human specimens of adult and pediatric BSG. Positive in situ hybridization signals are visualized in red, while blue depicts DAPI nuclear stain (original magnification, $\mathrm{x} 400$ ).

10 glioblastoma (GBM) cell lines and nine freshly resected GBM samples and observed a consistent pattern of miRNAs whose expression differs from surrounding normal brain tissue. However, most of these data were based on the analyses of supratentorial gliomas. BSG has not been genetically well studied, not to mention from the perspective of distinction between BSG heterogeneity. On the other hand, tumor location could be associated with specific tumorigenic pathway even within one histological entity (26). 
In the present study, a set of miRNAs were identified as differentially expressed in pediatric BSG compared to adult type, suggesting that altered miRNA expression may be involved in the acquisition of malignant progression of pediatric BSG. If we set 3.5-fold as the threshold of a differential expression level, the number of miRNAs which have at least 3.5-fold changes is not large. After data analysis, we found that, among the differentially expressed miRNAs, the most representative were miR-20a and miR-106b, with strong enrichment in pediatric BSG.

The development of satisfactory experimental models for simulating BSG heterogeneity is critical to our understanding of this tumor and the future discovery of novel therapies, for it could be adapted to facilitate experimental intervention and pre-clinical testing of new therapies $(10,27)$. Our previous models using C6 rat glioma cell lines have been reported (10). A potential limitation of rodent tumor-derived systems is that the response of rodent cell lines to treatment with anticancer agents can differ from that of human glioma cells (28). In the present study, altering of human glioma cell line offers a distinct advantage with regard to this concern (27). Furthermore, to verify our above observations in BSG models that reflected molecular distinction between two types of BSGs, we analyzed miR-20a and miR-106b expression in tissue sections from BSG patients, and found that the results were accordant. We presume that miR-20a and miR-106b could have putative causative involvement of acquisition of malignant progression of pediatric BSG.

Noteworthy, miR-106b belongs to miR-106b-25 cluster, whereas its paralog miR-17-92 cluster contains miR-20a. In these two miRNA families, the homologue miRNAs relations are: miR-106b matches miR-17-5p sequence, miR-93 matches miR-20a, and miR-25 matches miR-92a-1 (29). Accordingly, there may exist a coordination mechanism involving miR106b-25 and miR-17-92 clusters in BSG. The miR-106b-25 and miR-17-92 synergistic control apoptosis is supported by the work of Petrocca and colleagues, triggering a positive feedback loop that would support MYC, E2F1, and miR106b-25/miR-17-92 elevated expression while inactivating the TGF $\alpha$ pathway (29). Also, both miR-106b-25/miR-17-92 could directly target $\mathrm{p} 21 / \mathrm{CDKN} 1 \mathrm{~A}$, so as to contribute to tumor cell proliferation in part by regulating cell cycle progression and by modulating checkpoint functions $(30,31)$. Thus, it is of interest to note that miR-106b-25 cluster is present in selfrenewing neural stem/progenitor cells (NSPCs) and does not change its expression when cells are stimulated to undergo differentiation (32). miR-20a was identified to be involved in spontaneously progression of primary WHO grade II gliomas to WHO grade IV secondary glioblastomas (33), while our previous study observed that miR-106b were overexpressed in five glioblastoma cell lines (34).

The lack of knowledge on miRNA target genes hampers the full understanding on the biological functions of miRNAs. The target genes of miR-20a and miR-106b could be extracted using databases such as TargetScan, and PicTar, which provide a composite prediction of target genes from target prediction tools. Further studies are required to clarify the role of these genes in cellular biology. Besides, our previous finding indicated that the growth pattern and invasiveness of pediatric BSG could depend not only on the internal mechanism of the tumor cells, but also on the host cellular environment created for the tumor to spread into the adjacent brain tissue (10). The acquisition of malignant progression of pediatric BSG compared to adult patients could be attributed to the collaborative interaction of aberrantly expressed miRNAs of tumor cells (such as miR-20a and miR-106b) and the host cellular environment, which warrants further investigation.

In conclusion, our results demonstrate that miR-20a and miR-106b, at least in part, may play a role in acquired malignant progression in pediatric BSG compared with the adult group. The elucidation of the oncogenic role of these miRNA in modulating gene expression will present a comprehensive perspective for understanding the molecular bases and initiation of BSG heterogeneity. Antagonizing the corresponding miRNAs, thus may represent a possible antitumor approach for integrated cancer therapy to alter the gloomy outlook in pediatric BSG.

\section{Acknowledgements}

This study was supported by the China National Natural Scientific Found $(81172399,30872647)$, and the Beijing Natural Science Found (7092041).

\section{References}

1. Hargrave D, Bartels U and Bouffet E: Diffuse brainstem glioma in children: critical review of clinical trials. Lancet Oncol 7: 241-248, 2006.

2. Laigle-Donadey F, Doz F and Delattre JY: Brainstem gliomas in children and adults. Curr Opin Oncol 20: 662-667, 2008.

3. Guillamo JS, Doz F and Delattre JY: Brain stem gliomas. Curr Opin Neurol 14: 711-715, 2001.

4. Mischel PS and Cloughesy TF: Targeted molecular therapy of GBM. Brain Pathol 13: 52-61, 2003.

5. Hwang JH, Smith CA, Salhia B and Rutka JT: The role of fascin in the migration and invasiveness of malignant glioma cells. Neoplasia 10: 149-159, 2008

6. Guillamo JS, Monjour A, Taillandier L, Devaux B, Varlet P, Haie-Meder C, Defer GL, Maison P, Mazeron JJ, Cornu P and Delattre JY: Brainstem gliomas in adults: prognostic factors and classification. Brain 124: 2528-2539, 2001.

7. Mursch K, Halatsch ME, Markakis E and Behnke-Mursch J: Intrinsic brainstem tumours in adults: results of microneurosurgical treatment of 16 consecutive patients. Br J Neurosurg 19: 128-136, 2005.

8. Kesari S, Kim RS, Markos V, Drappatz J, Wen PY and Pruitt AA: Prognostic factors in adult brainstem gliomas: a multicenter, retrospective analysis of 101 cases. J Neurooncol 88: 175-183, 2008.

9. Epstein F and Wisoff JH: Surgical management of brain stem tumors of childhood and adolescence. Neurosurg Clin N Am 1: 111-121, 1990.

10. Liu Q, Liu R, Kashyap MV, Agarwal R, Shi X, Wang CC and Yang SH: Brainstem glioma progression in juvenile and adult rats. J Neurosurg 109: 849-855, 2008.

11. Gilbertson RJ, Hill DA, Hernan R, Kocak M, Geyer R, Olson J, Gajjar A, Rush L, Hamilton RL, Finkelstein SD and Pollack IF: ERBB1 is amplified and overexpressed in high-grade diffusely infiltrative pediatric brain stem glioma. Clin Cancer Res 9: 3620-3624, 2003.

12. Lee RC, Feinbaum RL and Ambros V: The C. elegans heterochronic gene lin-4 encodes small RNAs with antisense complementarity to lin-14. Cell 75: 843-854, 1993.

13. Karginov FV, Conaco C, Xuan Z, Schmidt BH, Parker JS, Mandel $G$ and Hannon GJ: A biochemical approach to identifying microRNA targets. Proc Natl Acad Sci USA 104: 19291-19296, 2007.

14. Cui Q, Yu Z, Purisima EO and Wang E: Principles of microRNA regulation of a human cellular signaling network. Mol Syst Biol 2: 46, 2006. 
15. Wang X, Han L, Zhang A, Wang G, Jia Z, Yang Y, Yue X, $\mathrm{Pu} P$, Shen $\mathrm{C}$ and Kang C: Adenovirus-mediated shRNAs for co-repression of miR-221 and miR-222 expression and function in glioblastoma cells. Oncol Rep 25: 97-105, 2011.

16. Bhatti I, Lee A, Lund J and Larvin M: Small RNA: a large contributor to carcinogenesis? J Gastrointest Surg 13: 1379-1388, 2009.

17. Song YJ, Tian XB, Zhang S, Zhang YX, Li X, Li D, Cheng Y, Zhang JN, Kang CS and Zhao W: Temporal lobe epilepsy induces differential expression of hippocampal miRNAs including let-7e and miR-23a/b. Brain Res 1387: 134-140, 2011.

18. Selvapandian S, Rajshekhar V and Chandy MJ: Brainstem glioma: comparative study of clinico-radiological presentation, pathology and outcome in children and adults. Acta Neurochir 141: 721-727, 1999.

19 Jallo G: Brainstem gliomas. Childs Nerv Syst 22: 1-2, 2006.

20 Salmaggi A, Fariselli L, Milanesi I, Lamperti E, Silvani A, Bizzi A, Maccagnano E, Trevisan E, Laguzzi E, Ruda R, et al: Natural history and management of brainstem gliomas in adults. A retrospective Italian study. J Neurol 255: 171-177, 2008.

21. Yin LX, Li DZ, Zhang JT, Wu Z and Zhang LW: Comparison of clinical characteristics and surgical outcomes between adults and children with astrocytomas of brain stem. Zhong Guo Wei Qin Xi Shen Jing Wai Ke Za Zhi 15: 158-160, 2010.

22. Pang JC, Kwok WK, Chen Z and Ng HK: Oncogenic role of microRNAs in brain tumors. Acta Neuropathol 117: 599-611, 2009.

23. Bottoni A, Zatelli MC, Ferracin M, Tagliati F, Piccin D, Vignali C, Calin GA, Negrini M, Croce CM and Degli Uberti EC: Identification of differentially expressed microRNAs by microarray: a possible role for microRNA genes in pituitary adenomas. J Cell Physiol 210: 370-377, 2007.

24. Gokhale A, Kunder R, Goel A, Sarin R, Moiyadi A, Shenoy A, Mamidipally C, Noronha S, Kannan S and Shirsat NV: Distinctive microRNA signature of medulloblastomas associated with the WNT signaling pathway. J Cancer Res Ther 6: 521-529, 2010.

25. Ciafre SA, Galardi S, Mangiola A, Ferracin M, Liu CG, Sabatino G, Negrini M, Maira G, Croce CM and Farace MG: Extensive modulation of a set of microRNAs in primary glioblastoma. Biochem Biophys Res Commun 334: 1351-1358, 2005.
26. Miwa T, Hirose Y, Sasaki H, Ikeda E, Yoshida K and Kawase T: Genetic characterization of adult infratentorial gliomas. J Neurooncol 91: 251-255, 2009.

27. Hashizume R, Ozawa T, Dinca EB, Banerjee A, Prados MD, James CD and Gupta N: A human brainstem glioma xenograft model enabled for bioluminescence imaging. J Neurooncol 96: 151-159, 2010.

28. Yaz G, Kabadere S, Oztopcu P, Durmaz R and Uyar R: Comparison of the antiproliferative properties of antiestrogenic drugs (nafoxidine and clomiphene) on glioma cells in vitro. Am J Clin Oncol 27: 384-388, 2004.

29. Petrocca F, Vecchione A and Croce CM: Emerging role of miR-106b-25/miR-17-92 clusters in the control of transforming grow th factor beta signaling. Cancer Res 68: 8191-8194, 2008.

30 Ivanovska I, Ball AS, Diaz RL, Magnus JF, Kibukawa M, Schelter JM, Kobayashi SV, Lim L, Burchard J, Jackson AL, et al: MicroRNAs in the miR-106b family regulate p21/CDKN1A and promote cell cycle progression. Mol Cell Biol 28: 2167-2174, 2008.

31. Inomata M, Tagawa H, Guo YM, Kameoka $\mathrm{Y}$, Takahashi $\mathrm{N}$ and Sawada K: MicroRNA-17-92 down-regulates expression of distinct targets in different B-cell lymphoma subtypes. Blood 113: 396-402, 2009

32. Peck B, Schulze A, Oliveras-Ferraros C, Vellon L, Joven J and Vazquez-Martin A: A role for the cancer-associated miR-106b 25 cluster in neuronal stem cells. Aging (Albany NY) 3: 329-331, 2011.

33. Malzkorn B, Wolter M, Liesenberg F, Grzendowski M, Stuhler K, Meyer HE and Reifenberger G: Identification and functional characterization of microRNAs involved in the malignant progression of gliomas. Brain Pathol 20: 539-550, 2010.

34. Zhou X, Ren Y, Moore L, Mei M, You Y, Xu P, Wang B, Wang G, Jia Z, Pu P, Zhang W and Kang C: Downregulation of miR-21 inhibits EGFR pathway and suppresses the growth of human glioblastoma cells independent of PTEN status. Lab Invest 90: $144-155,2010$ 